\title{
Die Energiewende
}

\section{Ein Echtzeitexperiment, das keinen Fehler erlaubt, oder eine große Chance für die Wirtschaft?}

\author{
DOI 10.1007/s11576-012-0327-1
}

\section{Die Autoren}

Prof. Dr. Hans Ulrich Buhl ( $\varangle)$

FIM Kernkompetenzzentrum Finanz-

\& Informationsmanagement

Universität Augsburg

Universitätsstraße 12

86159 Augsburg

Deutschland

hans-ulrich.buhl@wiwi.uni-augsburg. de

Prof. Dr. Michael Weinhold

Chief Technology Officer (CTO) der

Stabsabteilung Technik und

Innovation im Siemens Energy

Sector

Siemens AG

Wittelsbacher Platz 2

80333 München

Deutschland

michael.g.weinhold@siemens.com

Online publiziert: 2012-05-30

This article is also available in English via http://www.springerlink.com and http://www.bise-journal.org: Buhl HU, Weinhold M (2012) The Energy Turnaround. A Real-Time Experiment Allowing No Failure or a Major Opportunity for Our Economy? Bus Inf Syst Eng. doi: 10.1007/s12599-012-0221-9.

(C) Gabler Verlag 2012
Als es im März 2011 im japanischen Fukushima in Folge eines Tsunamis zu der verheerenden Reaktorkatastrophe kam, konnte man noch nicht ahnen, welche Auswirkungen dies auf die Zukunft der Energieversorgung haben würde. So haben viele Länder seitdem ihre Aktivitäten zur Realisierung einer Energiewende, also einem Wechsel von der Versorgung mit Kernenergie und fossilen Brennstoffen zu einer nachhaltigeren Energieversorgung unter vermehrtem Einsatz regenerativer Energien wie Wind oder Sonne, stark intensiviert: Während 2005 lediglich 55 Staaten über politische Ziele zum Ausbau erneuerbarer Energien verfügten, sind es mittlerweile bereits weltweit rund 190 Staaten. Auch in den Köpfen der Menschen macht sich ein Umdenken bemerkbar. So befürworteten in Frankreich Anfang 2012 bereits über 70 \% der Bevölkerung einen Atomausstieg - und das, obwohl Frankreich Ende 2010 mit einem Anteil von 75 \% Kernenergie an der gesamten Stromerzeugung weltweiter Spitzenreiter in dieser Hinsicht war. Einen kompromisslosen Ansatz verfolgt Deutschland, wo der Bundestag kurz nach der Reaktorkatastrophe in Japan den vollständigen Atomausstieg bis 2022 beschlossen hat, obwohl die wirtschaftlich sehr erfolgreichen Bundesländer im Süden des Landes bis dahin mehr als $50 \%$ ihres Strombedarfes mit Kernenergie gedeckt haben. Was aktuell abgeschwächt auch in anderen Ländern zu beobachten ist, muss - um im Jargon der Wirtschaftsinformatik zu bleiben - mehr „Revolution“ als „Evolution“ genannt werden. Eine solche bringt Risiken, aber auch Chancen mit sich. Nachfolgend soll aufgezeigt werden, dass die Energiewende eine Vielzahl von Chancen mit sich bringt - wenn man sie nur sinnvoll und konsequent mit einem durchdachten Plan angeht.

Zunächst einmal sei jedoch darauf hingewiesen, dass trotz aller „Revolution“ die Energiewende ein aktuelles, aber an sich kein neues Thema ist. Auch die WIRTSCHAFTSINFORMATIK hat dieses Thema schon sehr früh aufgegriffen. Bspw. haben wir bereits in der Ausgabe 4/2008 die enormen Potenziale der IT hinsichtlich der Energiewende über den Einsatz von Green IT hinaus aufgezeigt. Genau ein Jahr später wurde in der Ausgabe 4/2009 auf die Verantwortung der Wirtschaftsinformatik für unseren Planeten und die Gefahr einer bevorstehenden Ressourcenkrise hingewiesen. Im Editorial 1/2012 und dem zugehörigen Themenheft lag der Fokus dann auf der Thematik „Smart Grids“ und deren Rolle bei der Energiewende. Dies zeigt, dass die internationale Community durchaus schon lange vor Fukushima die Relevanz der Energiewende erkannt hat. Dies allein genügt aber nicht. Vielmehr muss für den Erfolg der Energiewende verhindert werden, dass einzelne Länder in blinden Aktionismus verfallen. Dies ist gerade vor dem Hintergrund wichtig, dass die mit der Energiewende verbundenen Ziele wie die verstärkte Nutzung regenerativer Energien (bspw. soll in Deutschland deren Anteil am elektrischen Energieverbrauch von $20 \%$ im Jahr 2011 auf mindestens $35 \%$ im Jahr 2020 ansteigen) mit hohen Herausforderungen verbunden sind. Einige dieser Herausforderungen werden im Folgenden knapp skizziert:

- Die Ernte einiger erneuerbaren Energien ist schwer plan- und steuerbar - man denke hier bspw. an Wind- oder Sonnenenergie, die nur bei entsprechenden Wetterlagen Strom erzeugen. Dies führt häufig unweigerlich zu einem Mismatch zwischen Gewinnungs- und Nachfragezeiten.

- Zur Absicherung gegen solche Versorgungsrisiken sind Energiespeicher oder konventionelle Kraftwerke nötig. Bauvorhaben für Speicher stehen jedoch teilweise in Konflikt mit geltenden Vorgaben zum Naturschutz oder sind in der Bevölkerung unbeliebt. Außerdem befinden sich in diesem Bereich viele Konzepte noch in der Forschungs- oder Entwicklungsphase und können daher aktuell noch nicht kommerziell eingesetzt werden. Bauprojekte für konventionelle Kraftwerke erfahren ebenfalls häufig lokalen Widerstand. 
- Auch Onshore-Windanlagen werden aufgrund der damit verbundenen Einschnitte in die Natur in der Bevölkerung zunehmend kritisch hinterfragt.

- Für den Transport von erneuerbarer Energie ist ein massiver Ausbau des Höchstspannungsnetzes sowohl auf nationaler als auch internationaler Ebene nötig. Allein in Deutschland müssten bis 2015 rund 400 Kilometer des bestehenden Netzes verstärkt und weitere 850 Kilometer neu gebaut werden. Eine Herausforderung ist dies insbesondere vor dem Hintergrund, dass dort im Zeitraum von 2006 bis 2011 gerade einmal 90 Kilometer neu errichtet wurden. Weitere $3600 \mathrm{~km}$ an Neubaubedarf sieht die dena-Netzstudie II bis 2020. Auch das Netz von Kuppelleitungen zwischen den verschiedenen EU-Ländern muss erheblich ausgebaut werden.

- Nicht außer Acht gelassen werden darf die Tatsache, dass für die Herstellung der für erneuerbare Energien benötigten Infrastruktur teils in nennenswertem Umfang knappe nicht-energetische Ressourcen benötigt werden. Als knappes Gut auf dem Weltmarkt zeigen sich seltene Erden wie Neodym, die beispielsweise bei Windturbinen zum Einsatz kommen. An dieser Stelle muss verhindert werden, dass die Energiewende als Brandbeschleuniger für eine sich anbahnende Ressourcenkrise in Bezug auf nicht-energetische Ressourcen wirkt.

- Neben all dem ist zu beachten, dass die Energiewende so gestaltet werden muss, dass sie nicht die weltweiten Klimaschutzziele gefährdet. So war bspw. in Deutschland nach der Stilllegung einiger Kernkraftwerke im Jahr 2011 zu beobachten, dass viele Energieanbieter den Wegfall der Kernenergie dadurch kompensierten, dass sie die Laufzeit ineffizienter und emissionsstarker Kohlekraftwerke verlängerten oder gar bereits stillgelegte Altanlagen wieder in Betrieb nahmen. Dies ist vor allem deshalb kritisch zu betrachten, weil bereits 2010 der weltweite $\mathrm{CO}_{2}$-Austoss sowie die $\mathrm{CO}_{2}$ Konzentration in der Atmosphäre auf jeweils neue Rekordwerte anstiegen.

All diese Herausforderungen können nur mithilfe eines international koordinierten und flexiblen Masterplans bewältigt werden. So muss dringend festgelegt werden, wo welche Kraftwerke benötigt werden, um die Grundlast zu sichern und damit Stromengpässe zu vermeiden. Zudem muss geplant werden, wo und auf welche Art und Weise Stromnetze ausgebaut werden müssen. Diese Planung muss über nationale Grenzen hinweg geschehen, um damit das Problem der vielen existierenden Partikularinteressen zu reduzieren. Darüber hinaus darf ein Masterplan jedoch kein statisches Konstrukt sein, sondern stattdessen über überprüfbare Meilensteine verfügen, um flexibel auf bspw. neue Technologien oder Preisentwicklungen adaptierbar zu sein.

Was kann passieren, wenn ein solcher Masterplan - wie es derzeit der Fall ist - nicht existiert? Zum Beispiel werden regenerative Energien auch an ertragsschwachen Standorten installiert. So betrug im Jahr 2010 die installierte Leistung der Solaranlagen in Nordrhein-Westfalen (jener Region in Deutschland mit der im langjährigen Durchschnitt geringsten Sonnenscheindauer) 1.941 MW. Allerdings ist für die Installation von erneuerbaren Energien an den besten Standorten oft ein Netzausbau erforderlich, da die Erzeugung dann oft nicht in der Nähe der Lastzentren liegt. Aktuell scheitert man aufgrund fehlender Stromtrassen bereits am Transport von in Norddeutschland erzeugtem Windstrom in Verbrauchszentren wie bspw. im Süden des Landes. Weitere Steine auf dem Weg zur erfolgreichen Energiewende stellen unklare politische Rahmenbedingungen dar, die zu einer mangelnden Planbarkeit für Industrie und Privathaushalte führen. Als Folge werden die für eine erfolgreiche Energiewende nötigen Investitionen bspw. in neue Kraftwerke, energiesparende Produktionsanlagen und Prozesse sowie für eine Umrüstung privater Haushalte zur Nutzung von Smart Grids zu häufig noch sowohl von Energieanbietern als auch -nachfragern gescheut statt forciert. Umso wichtiger ist es daher, dass die Politik klare Signale setzt und dadurch Planungssicherheit bei Investoren schafft und die Investitionsbereitschaft ankurbelt.

Durch einen fehlenden Masterplan macht sich ein Land wie Deutschland selbst und ohne Not zum Echtzeitexperiment, welches im Ausland oft mit Verwunderung (Internationale Atomenergie-Organisation) aufgenommen wird. Problematisch ist dabei, dass das Experiment nicht in einem Labor stattfindet, sondern bereits scharfgeschaltet ist. Das Resultat ist: Man darf sich keinen Fehler erlauben, da die Folgen verheerend sein könnten. Sollte es bspw. nicht gelingen, die Mindestversorgung an Energie aufrecht zu erhalten, drohen Blackouts mit enormen Auswirkungen (der größte Stromausfall in der Geschichte Nordamerikas verursachte einen wirtschaftlichen Schaden von über 6 Milliarden US-Dollar). Außerdem haben gestiegene Strompreise bereits in der Vergangenheit zu einer Abwanderung von Unternehmen ins Ausland beigetragen. Als prominentes Beispiel lässt sich hier BMW aufführen, die ihre (energieintensive) Produktion 
von Karbonfasern für Elektroautos von Deutschland in die USA verlagerten. Für eine Volkswirtschaft führt dies zu einem Verlust von Arbeitsplätzen, Know-how und Steuereinnahmen.

Anstatt nun aber vor diesen Herausforderungen und damit verbundenen Risiken zu kapitulieren und den Erfolg der Energiewende abzuschreiben, sollten wir uns jedoch lieber auf die enormen Chancen konzentrieren, welche die Energiewende mit sich bringt. So ist es zwar hinsichtlich des weltweiten $\mathrm{CO}_{2}$-Austoßes und des Klimawandels nicht alleinig ausschlaggebend, was in einem einzelnen Land passiert, aber es kann eine massive Signalwirkung für die Welt haben. Und die sich daraus ergebenden Chancen gilt es zu nutzen!

Bleiben wir beim Beispiel Deutschland: Wenn die Energiewende dort erfolgreich und dabei gleichzeitig auch bezahlbar gelingt und Deutschland der Welt als Modell für einen erfolgreichen Energiewechsel dienen kann, wird mit hoher Wahrscheinlichkeit auf diese Weise ein Innovationsfeuerwerk ausgelöst, das in einen erheblichen Wachstumsschub für die Wirtschaft mündet. Diese Hypothese lässt sich schon allein darauf stützen, dass sich die deutsche Wirtschaft bereits in der Vergangenheit durch eine exzellente Anpassungsfähigkeit ausgezeichnet hat. So hat auch der hohe Ölpreis dem Automobilland Deutschland nicht nachhaltig geschadet, sondern vielmehr die Kreativität der Automobilbranche angeregt und damit zu einer Weiterentwicklung von effizienten Antriebsformen geführt. Analog hierzu sollten wir auch das hohe Innovationspotenzial, das hinter der Energiewende steckt, als Großchance für die Wirtschaft sehen. Bereits jetzt gibt es eine Vielzahl von Innovationen, die bereits in aller Munde sind - zu nennen sind hier bspw. Technologien zur effizienten Energiesteuerung wie Smart Meters, moderne Solar- und Antriebstechniken, Maschinen oder neue Baustoffe, die zu einer höheren Energieeffizienz führen. Eine Erhöhung der Effizienz im gesamten System, von der Stromerzeugung über den Transport, die Verteilung und den Verbrauch ist generell ein Schlüsselelement auf dem Weg zu einem nachhaltigeren Energiesystem. Ziel ist dabei nicht unbedingt eine Effizienzsteigerung bis zum technisch machbaren Grad, vielmehr zum ökonomisch sinnvollen Level. Hier gibt es noch viel Raum für Innovationen.

Enormes Innovationspotenzial liefert zudem die simultane Optimierung der Verbindung von Strom, Wärme und Gas - einem der wichtigsten Hebel der Energiewende. Bei einer Einspeisung von Windstrom in die Raumwärmeversorgung könnte man den Wärmebedarf von großen Teilen der Gebäude (die immerhin für mehr als $30 \%$ des Primärenergieeinsatzes stehen) sichern und den $\mathrm{CO}_{2}$-Ausstoß weiter verringern. Dedizierte Wärmespeicher, auch lokal in Haushalten in Form von Heißwassertanks, könnten das elektrische System generell gut ergänzen. Denn Schwankungen im Stromaufkommen können mithilfe thermischer Speicher gut gepuffert werden. Umgekehrt bieten in gut wärmegedämmten Häusern auch effiziente elektrische Heizungen, bspw. in Form von Wärmepumpen, Potenzial für eine weitergehende Verzahnung von Strom und Wärme. Wärmepumpen in Niedrigenergiehäusern sind zunehmend verbreitet und die Einbindung des Fernwärmesystems ins Stromnetz wird derzeit bereits diskutiert und untersucht.

Das ist jedoch nicht alles. Ein Paradebeispiel für die Innovationswirkung der Energiewende stellt die hocheffiziente Wasserstoff-Elektrolyse mithilfe der PEM-Technologie dar, die von Siemens aktuell entwickelt und getestet wird. Die Idee hinter dieser Technologie, die bis 2015 Marktreife erlangen soll, ist an sich simpel: Man verwendet überschüssigen Strom aus erneuerbaren Energien und erzeugt daraus Wasserstoff. Dieser lässt sich in der Chemieindustrie direkt verbrauchen; dort gibt es auch bereits Wasserstoffnetze. Er könnte auch für Mobilitätszwecke genutzt werden und der Brennstoffzelle zum Durchbruch verhelfen. Zudem lässt sich Wasserstoff auch als Beimischung direkt ins Erdgasnetz einspeisen. In einem weiteren chemischen Prozess ließe sich aus Wasserstoff sogar, falls erforderlich, synthetisches Erdgas erzeugen. Im deutschen Gasnetz lassen sich bis zu 200 Terawattstunden Energie speichern, was dem Dreifachen der hiesigen Wind- und Solarproduktion des Jahres 2011 entspricht. Die positiven Effekte davon liegen auf der Hand: Zum einen wird das bereits beschriebene Problem des Mismatchs der Gewinnungs- und Nachfragezeiten von erneuerbaren Energien umgangen. Bspw. kann dann Windenergie, die nachts bei geringer Energienachfrage gewonnen wird, gespeichert und später verwendet werden. Zum anderen liegt nahe, dass ein Unternehmen, welches eine solche Technologie entwickelt, auch wirtschaftlich davon profitiert: Der Siemens-Konzern, welcher jährlich eine Milliarde Euro in die Entwicklung neuer Technologien für die Energiewirtschaft investiert, sieht derzeit das stärkste 
Wachstum im Energiebereich. Da Energieversorger mit solchen innovativen Speichermethoden zukünftig angebotsinduzierte Schwankungen des Strompreises (bspw. Preiseinbrüche bei Überkapazität) verringern können, ist es auch naheliegend, dass eine solche Technologie international auf Anklang stoßen wird.

Auch die Entwicklung einer „schlüsselfertigen“ Lösung zum Bau eines europäischen Verbindungsnetzes bietet hohe wirtschaftliche Potenziale. Die dazu notwendige Technologie - die sogenannte Hochspannungsgleichstromübertragung (HGÜ-Verbindung) existiert bereits und wird bspw. in China in der besonders hohen und damit verlustarmen Spannungsebene von 800.000 Volt seit 2009 eingesetzt. Eine europaweite Verwendung dieser Technologie, zum Beispiel auf der Spannungsebene 500 Kilovolt, würde ebenfalls für volle Auftragsbücher bei den Technologiekonzernen sorgen, da der globale Markt für Stromübertragungen insgesamt in den nächsten fünf Jahren zwischen fünf und neun Milliarden Euro pro Jahr liegen wird. Der darin enthaltene Markt für HGÜ-Verbindungen wird sich den Schätzungen von Siemens zufolge von derzeit drei Milliarden Euro pro Jahr im selben Zeitraum verdoppeln, da die Nachfrage nach HGÜVerbindungen rasant ansteigt.

Selbst die auf den ersten Blick kritisch zu sehende Kürzung der Solarförderung 2012 mit stärkeren Eigenverbrauchsvorgaben kann positive Impulse für die Wirtschaft mit sich bringen: Dadurch, dass es finanziell vorteilhaft ist, seinen Eigenverbrauch an mit Solarenergie gewonnenem Strom zu erhöhen, wird es wichtig, Gewinnung und Verbrauch von Solarstrom zeitlich stärker zur Deckung zu bringen.

Wie kann nun die Wirtschaftsinformatik dazu beitragen, diese enormen Potenziale zu nutzen? Zum einen muss die Vielzahl der dezentralen Stromerzeuger störungsfrei koordiniert werden. Hierzu ist im Verteilernetz die entsprechende Intelligenz nötig (Smart Grid). Diese Intelligenz könnte bspw. durch die Verwendung von Agentensystemen generiert werden, welche durch richtigen Einsatz auch den Verbrauch im Verteilernetz steuern könnten, um damit Erzeugungsspitzen und Flautezeiten abzufedern. Generell wird es in Zukunft immer wichtiger werden, in Echtzeit auf Angebot und Nachfrage zu reagieren. Hier spielt die Wirtschaftsinformatik eine zentrale Rolle. Die Energieversorger haben dies erkannt und wissen, dass in deren Branche ohne IT nichts mehr geht. Man denke hier bspw. an lernende Business-Analytics-Programme, die für Energiehändler Auktionsergebnisse auswerten und dabei Einflussfaktoren wie Wetterprognosen oder Wartungsintervalle von Kraftwerken berücksichtigen. Der deutsche Energiekonzern Vattenfall gibt zum Beispiel an, fünf bis zehn Prozent mehr Zuschläge bei Energieauktionen zu bekommen als bei Regelenergie-Auktionen ohne ITUnterstützung.

Darüber hinaus können auch wir innerhalb der internationalen Community unseren Beitrag zum erfolgreichen Gelingen der Energiewende leisten. Zum einen haben Themen in Bezug auf den nachhaltigen Umgang mit knappen Ressourcen - seien sie nun energetisch oder nicht-energetisch - noch immer einen Randplatz in den Lehrprogrammen unserer Hochschulen. Hier liegt es an uns, Führungskräfte von morgen mit dem nötigen Fachwissen auszustatten. Zudem könnten regelmäßige NachhaltigkeitsRubriken in Zeitschriften etabliert werden.

Was lässt sich zusammenfassend sagen?

Die Energiewende bringt eine Vielzahl von Herausforderungen mit sich, zu deren Bewältigung ein Masterplan nötig ist. Dieser muss grenzübergreifend und flexibel gestaltet sein und ein koordiniertes Vorgehen bei der Energiewende sicherstellen. Bei der Erstellung eines solchen Masterplans ist vor allem die Politik gefragt. Aber auch Energieversorger, Netzbetreiber, Technik- und Baukonzerne, Kabelhersteller und nicht zuletzt die IT-Industrie müssen ihren Beitrag leisten. Wichtig ist hier jedoch die verstärkte Einbindung und Unterstützung sowohl der Politik als auch der Wissenschaft. Die Wirtschaftsinformatik ist hierbei gerufen, einen Koordinations- und Integrationsbeitrag zu leisten.

Neben dem Masterplan muss aber letztlich auch die Bevölkerung zur Energiewende beitragen. Wir alle brauchen deshalb ein Bewusstsein dafür, dass eine erfolgreiche Energiewende zwar den Beitrag jedes Einzelnen erfordert, zugleich aber nur mit Plan und nicht von heute auf morgen funktionieren kann. Nationale oder gar regionale Insellösungen reichen nicht, wir brauchen internationale Kooperation. Wenn dieses Bewusstsein wächst und mit Engagement implementiert wird, dann kann dieses Echtzeitexperiment zu einem großen Erfolg werden! 